\title{
Nerve Growth Factor and Vascular Endothelial Growth Factor: Retrospective Analysis of 63 Patients with Salivary Adenoid Cystic Carcinoma
}

\author{
Hao $\mathrm{Li}^{1}$, Xiao-lin Nong ${ }^{1 *}$, Qi Chen ${ }^{1}$, Yi-ping Yang ${ }^{2}$, Jia-quan $\mathrm{Li}^{3}$, Yan-ning $\mathrm{Li}^{4}$ \\ ${ }^{1}$ Department of Oral and Maxillofacial Surgery, College of Stomatology, Guangxi Medical University, Nanning, China \\ ${ }^{2}$ Department of Pathology, Stomatological Hospital Affiliated to Guangxi Medical University, Nanning, China \\ ${ }^{3}$ Laboratory Center, Guangxi Medical University, Nanning, China \\ ${ }^{4}$ Department of Epidemiology and Health Statistics, College of Public Health, Guangxi Medical University, Nanning, China
}

\begin{abstract}
Aim To detect the expression of nerve growth factor (NGF) and vascular endothelial growth factor (VEGF) in salivary adenoid cystic carcinoma (SACC) tissues, as well as to determine the correlation between growth factor expression and prognosis in SACC.

Methodology Medical records of 63 patients surgically treated for SACC between January 1988 and October 2005 were reviewed. Immunohistochemistry was performed to examine the expression of NGF and VEGF in tumor tissues. Kaplan-Meier analysis and Cox's proportional hazard regression model were applied to assess predictors of survival.
\end{abstract}

Results NGF and VEGF were overexpressed in SACC tissues, compared with those in normal salivary tissues $(P<0.05)$, and the staining intensity of these two factors was stronger in groups of solid subtype, advanced TNM stage, perineural invasion and recurrence. Patients with highexpression of NGF and VEGF, solid subtype, advanced stage, perineural invasion, recurrence and extended resection alone had worse survival rates $(P<0.05)$.

Conclusion NGF and VEGF are expressed increasingly in the tissues of SACC cases with invasion and metastasis. NGF expression and VEGF expression are independent prognosis factors for survival.

Keywords nerve growth factor, vascular endothelial growth factor, salivary adenoid cystic carcinoma, prognosis

Received Oct. 15, 2009; Revision accepted Dec.1, 2009

\section{Introduction}

Salivary adenoid cystic carcinoma (SACC) is a malignant neoplasm that originates in both minor and major salivary glands, characterized by slow growth, diffuse invasion and potential to produce distant metastases, mainly to the lungs and bones (Umeda et al., 2002). Its peculiar biological behaviors always cause great difficulty in treatment and prognosis. Though the mechanism of its invasion and metastasis still remains undefined to some extent, evidence suggests that progression of SACC is affected by varied expression of some growth factors and their receptors, which also impact the prognosis of patients with SACC. It has been reported that there is an overexpression of transforming growth factor-alpha (TGF- $\alpha$ ) and epidermal growth factor receptor (EGFR) in adenoid cystic carcinoma (ACC), and that the level of EGFR is higher in the pathological subtype of greater malignancy and associated with worse prognosis (Chiang et al., 2001; Gibbons et al., 2001; Liu et al., 2007).

Nerve growth factor (NGF) belongs to the neurotrophic factor family. Not only can NGF modulate growth, development and regeneration of many types of nerve cells in the central and peripheral nervous system, but it can also 
influence proliferation and diffrentiation of tumors, including head and neck neoplasms (Sortino, et al., 2000; Zhu, et al., 2002; Kan, et al. 2002; Luo, et al., 2006). Recently, a few experiments have shown that NGF or its receptor is highly expressed in some malignancies with neurotropism such as prostatic carcinoma, and NGF is also specifically elevated in oral squamous cell carcinoma with perineural invasion (Fanburg-Smith and Miettinen, 2001; Fuminori, et al., 2001; Kolokythas et al. 2006), indicating that this factor is relevant to neurotropism. In addition, NGF overexpression is associated with lymph node metastasis, distant metastasis, higher TNM stage and lowered survival of patients with squamous cell carcinoma of head and neck (Tsunoda et al., 2006).

Vascular endothelial growth factor (VEGF) is the most potent mitogen of vascular endothelium. VEGF is able to promote differentiation, proliferation and migration of endothelial cells, stimulate neovascularization, and can induce invasion and metastasis of tumor cells (Witte et al., 1998; Gerwins et al., 2000). It has been shown that a variety of tumors, such as gastric carcinoma, express more VEGF than normal tissues. Among patients with malignancies, including head and neck squamous cell carcinomas, high VEGF expressors have a worse prognosis than low expressors (Maeda and Chung, 1996; Inoue et al., 1997; Burian et al., 1999; Ishigami et al., 1999; Mineta et al., 2000; Xu et al., 2004).

Though increased expression of NGF and VEGF has been demonstrated in the growth, development and regeneration of tumors often associated with perineural or perivascular invasion, and though these two factors have been suspected to play critical roles in the progression of SACC as well as in assessment of the prognosis, it has not been clarified how the two factors influence the invasion and metastasis of SACC. There also have not been any reports of their direct contribution to the prognosis of the patients. The objectives of our study were to review the medical data of 63 SACC treated in our hospital, to investigate NGF and VEGF expression in their tumour specimens, and to explore the relevance between the expression and the prognosis of SACCs, as well as the relationship to invasion and metastasis.

\section{Materials and Methods}

\section{Clinical data of SACC cases}

This retrospective study reviewed 63 SACC patients with a postoperative pathological diagnosis, who were treated in the Department of Oral and Maxillofacial Surgery, Stomatological Hospital affiliated to Guangxi Medical University, between January 1988 and October 2005. The included cases did not undergo preoperative radiotherapy, chemotherapy or other related anti-tumor therapies and they had specific information regarding onset of disease, time of termination and status of treatment, as well as the time and cause of death. Data was gathered on gender, age, site of origin, surgical margin status, pathological subtype, TNM classification, perineural invasion, recurrence, treatment modality, etc. There were 34 females and 29 males. The age range was from 23 to 69 years with an average age of 47 years. The cases of the 40 to 60 year olds accounted for $68.3 \%$. Of the 63 cases, 36 were identified with ACC of the major salivary glands ( 9 originated from the parotid gland, 12 from the submandibular gland, and 15 from the sublingual gland); 27 were identified with ACC of the minor salivary glands (11 originated from the palate, 7 from the cheek, 5 from the body of the tongue, 3 from the base of tongue, and 1 from the retromolar pad). All of the surgical margins were negative. In these 63 cases, there were 44 of cribriform-tubular subtype and 19 of solid subtype, according to the guidelines of World Health Organization (Barnes et al., 2005); 11 of stage I , 25 of stage II, 19 of stage III, and 8 of stage IV, according to the TNM staging system of International Union Against Cancer (Sobin et al., 2010). The cases of stages I and II were categorized as an early-stage group, and those of stages III and IV as the advanced-stage group. Meanwhile, these cases were classified into either perineural-invasion or nonperineuralinvasion, according to whether they had nerveinvaded manifestations such as pain or facial paralysis. At the time of surgery a total of 5 cases had nervi facialis involvement, nervi lingualis involvement in 9, nervi hypoglossus involvement in 2, nervi palatinus anterior involvement in 1 , in 
each the nerves being surrounded or invaded by tumor cells. Cases included were also divided into recurrence or recurrence-free group, according to the results of postoperative biopsy and CT examination. In the patients studied, 28 underwent extended resection of primary tumor alone, 9 extended resection and ipsilateral modified neck dissection, 11 extended resection and postoperative radiotherapy ranging from 40 to $60 \mathrm{~Gy}$, and 15 extended resection and postoperative chemotherapy (5-fluorouracil + methotrexate + vincristine).

\section{Data of follow-up}

All durations were measured from the date of surgery to the date of dropout, SACC-related death or the last contact if the patient was still alive when the survival duration was calculated. The period of follow-up lasted 6 to 220 months with 31st Dec 2006 as the end.

\section{Immunohistochemistry of specimens}

All specimens were fixed with $4 \%$ formalin for 24 hours, embedded in paraffin, cut into $5 \mu \mathrm{m}$ thick sections, deparaffinized in xylene and hydrated through graded ethanol. Antigen retrieval was performed in $10 \%$ ethylene diaminetetraacetic acid (EDTA, pH 7.4) at $37^{\circ} \mathrm{C}$. Then, sections were sequentially incubated in $3 \% \mathrm{H}_{2} \mathrm{O}_{2}$ at room temperature for 10 minutes, washed with phoshphate buffered solution (PBS), and treated with goat serum at $37^{\circ} \mathrm{C}$ for 10 minutes, before they were incubated with the primary antibody (Maxim Biotech Co., China) at $4^{\circ} \mathrm{C}$ overnight. Later, these sections were incubated with the secondary antibody (Maxim Biotech Co., China) labeled by biotin for 10 minutes, washed with PBS, then incubated with anti-biotinylated protein-peroxidase complex at $37^{\circ} \mathrm{C}$ for 10 minutes, washed with PBS, detected with 3,3-diamino-benzidine (DAB; Maxim Biotech Co., China) as the chromogen, and finally stained with hematoxylin. Six (6) cases of normal salivary gland tissues served as normal control, a proved positive section as positive control, and PBS instead of the primary antibody as negative control. $\mathrm{DAB}$ staining was done similarly with these conditions.

\section{Immunohistochemical analysis}

The positive localization of NGF and VEGF was in the cytoplasm or cell membranes. The immunohistochemical analysis was scored with Volm's criteria as reference: 0 , cell staining similar to the background; 1, light yellow cytoplasm and staining of less than $25 \%$ of cells; 2 , brown cytoplasm and staining of $25 \%-50 \%$ of cells; 3 , brown particles in cytoplasm and staining of $50 \%-75 \%$ of cells; and 4 , dark brown particles in cytoplasm and staining of greater than $75 \%$ of cells (Volm et al., 1997). The staining intensity in scores $0-2$ was described as weak and that in scores $3 / 4$ as strong. The immunostaining was analyzed using pathological image analyzer (DMR + Q550, Leica, German), and evaluated independently by two pathologists who were blinded to clinical data. Scoring differences were discussed to reach consensus. All of the experiments were repeated three times.

\section{Statistic analysis}

Calculations were carried out with SPSS 13.0 (SPSS, USA). Chi-square Test and Student's $t$-test were used for single factor analysis to determine significance. The overall survival was estimated using the Kaplan-Meier method, and compared using the log-rank statistic. The probable factors affecting long-term survival of patients with SACC were analyzed using the Cox's proportional hazard regression model. $P<0.05$ was considered significant.

\section{Results}

\section{Expression of NGF in SACC tissues}

The positive rate of NGF in 63 SACC cases was $68.25 \%$ (43/63), and NGF was mainly detected in membranes or cytoplasm of carcinoma cells as well as some neurofibers. Fifteeen (15) cases showed strong staining in solid group (78.95\%), with stained locations scattered in carcinoma cell clusters, and 17 in cribriform-tubular group (38.64\%), with staining cells predominantly in adenoid areas. The immunoreaction in the solid group was stronger 


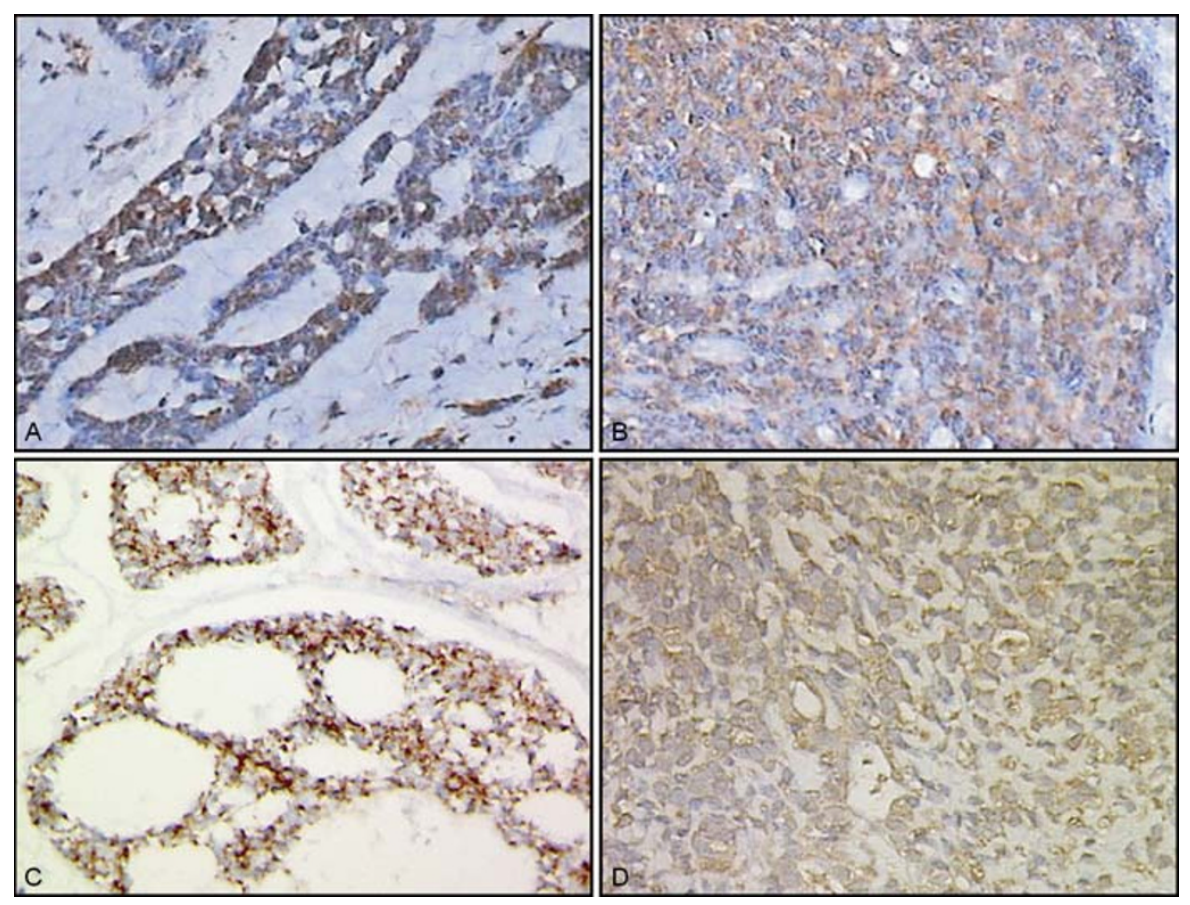

Figure 1 Expression of NGF and VEGF in SACC tissues (DAB Staining) $(\times 40)$

(A): NGF in the cribriform-tubular tissue. (B): NGF in the solid tissue. (C): VEGF in the cribriform-tubular tissue. (D): VEGF in the solid tissue.

than that in cribriform-tubular group. The strong staining rate was $82.35 \%(14 / 17)$ in the perineuralinvasion group and $39.13 \%(18 / 46)$ in the nonperineural-invasion group, with NGF expression in the former higher than in the latter (Figure 1). Moreover, the tumor cells around neurofibers were stained more intensely than those more distant, and had higher strong staining rates $(71.62 \%)$ than the latter $(39.18 \%)(P<0.05)$. In normal salivary gland tissues, NGF-immunoreactivity was weak in ductal cells and negative in acinous cells.

\section{Expression of VEGF in SACC tissues}

VEGF expression was observed in $79.37 \%$ of cases $(50 / 63)$ and was mainly localized in tumor cell cytoplasm and membranes, with medium staining in the endangium. The specimens of strong staining accounted for $22.72 \%(10 / 44)$ of the cribriform-tubular group and for $68.42 \%$ $(13 / 19)$ of the solid group; the immunoreactivity was more intense in the latter than the former $(P<0.05)$ (Figure 1). Eight (8) in perineural-invasion group overexpressed VEGF (47.06\%) with 9 in non-perineural-invasion group (19.56\%) doing so, and the expression was higher in the former group than that in the latter $(P<0.05)$. Little VEGF- immunoreactivity was seen in normal salivary glands.

\section{Comparison of expressions in different groups}

Student's $t$-test showed that in both NGF and VEGF immunostaining, there were more positive cells in groups of solid subtype, advanced stage, perineural-invasion and recurrence than in groups of cribriform-tubular subtype, early stage, nonperineural-invasion and recurrence-free (Tables 1,2).

Table 1 Percentage of NGF positive cells in SACC tissues $(\bar{x} \pm s)$

\begin{tabular}{lccc}
\hline \multicolumn{1}{c}{ Group } & $\begin{array}{c}\text { Percentage of } \\
\text { NGF positive cells }\end{array}$ & $t$ & $P$ \\
\hline Cribriform-tubular subtype & $20.21 \pm 8.57$ & & \\
Solid subtype & $29.94 \pm 7.69$ & 3.00 & 0.039 \\
Advanced-stage & $22.51 \pm 7.09$ & & \\
Early-stage & $10.66 \pm 8.28$ & 5.94 & 0.017 \\
Perineural-invasion & $27.50 \pm 9.04$ & & \\
Non-perineural-invasion & $18.73 \pm 7.66$ & 3.74 & 0.032 \\
Recurrence & $25.83 \pm 6.24$ & & \\
Recurrence-free & $18.42 \pm 7.18$ & 6.01 & 0.021 \\
\hline
\end{tabular}


Table 2 Percentage of VEGF positive cells in SACC tissues $(\bar{x} \pm s)$

\begin{tabular}{lccc}
\hline \multicolumn{1}{c}{ Group } & $\begin{array}{c}\text { Percentage of } \\
\text { VEGF positive cells }\end{array}$ & & \\
\hline Cribriform-tubular subtype & $22.17 \pm 7.00$ & & \\
Solid subtype & $31.14 \pm 6.14$ & 3.07 & 0.037 \\
Advanced-stage & $16.14 \pm 7.80$ & & \\
Early-stage & $11.51 \pm 9.61$ & 3.26 & 0.026 \\
Perineural-invasion & $26.78 \pm 8.85$ & & \\
Non-perineural-invasion & $17.48 \pm 7.43$ & 3.63 & 0.035 \\
Recurrence & $24.99 \pm 7.50$ & & \\
Recurrence-free & $19.82 \pm 8.25$ & 5.97 & 0.028 \\
\hline
\end{tabular}

\section{Analysis of follow-up}

There were complete follow-up data on for all the cases. At the end of the follow-up, 47 cases survived $(74.60 \%$ of 63$)$ with 16 dead $(25.40 \%)$. In the 16 deaths, 9 died of locoregional recurrence and invasion (56.25\%), 6 of pulmonary metastasis $(37.50 \%), 1$ of intracranial metastasis $(6.25 \%)$. The overall survival rates at 3 years, 5 years and 10 years were $77.64 \%, 68.31 \%$ and $39.26 \%$, respectively. The median survival was 128 months, ranging from 7 to 203 months.

\section{Analysis of single factors}

In 63 patients, 29 were male, and 34 were female. The postoperative survivals of males and females were not significantly different $(P>0.05)$.

The mean age of presentation in the series was 49 years, and ranged from 23 to 78 years of age. Age was not a significant predictor of survival $(P>0.05)$.

Patients whose tumor originated from the minor salivary gland appeared to have a worse survival $(P<0.05)$ and higher rate of perineural invasion which was seen in 8 of 17 cases $(47.06 \%)$. In addition, the survival of the cases with tumor origin from the parotid gland or submandibular gland was better than for those with origin from the sublingual gland, but the difference was not statistically significant $(P>0.05)$.

In the 16 deaths, there were 7 of the cribriformtubular (15.91\%), and $9(47.37 \%)$ of the solid group; the postoperative survival of the former was better $(P<0.05)$.
Of the 63 cases, at the time of diagnosis, 11 were at stage I (17.46\%), 25 at stage II (39.68\%), 19 at stage III $(30.16 \%)$ and 8 at stage IV (12.70\%). Among the 16 cancer-related deaths, there were 2 of stage I , 4 of stage II, 7 of stage III, 3 of stage IV. The survival of the advanced-stage was worse than that of the early-stage tumors $(P<0.05)$.

There were 17 patients in whom the tumor had invaded the nerve, and 11 of these $(64.71 \%)$ died of SACC. The cases with nerve invasion had a lower survival rate compared to those without nerve invasion $(P<0.05)$.

In the studied cases, 18 developed recurrence after surgery; 9 of these (50\%) dying of cancerrelated diseases. Their survival was lower than that of the recurrence-free $(P<0.05)$.

Among the 63 included patients, 28 underwent extended resection to remove the tumor en bloc, with 11 deaths in this group at the end of the follow-up; 9 accepted extended resection and ipsilateral modified neck dissection, with 4 deaths. Extended resection and postoperative radiotherapy or chemotherapy were performed to the remaining 26 cases, and 2 of these died of SACC. The ones with extended resection combined with other modalities had better survival than those with extended resection alone. However, no significant difference was seen among the survivals of the patients with ipsilateral modified neck dissection, postoperative radiotherapy or postoperative chemotherapy $(P<0.05)$.

The patients exhibiting low NGF expression had better survival than those exhibiting high expression $(P<0.05)$. Moreover, VEGF also had an impact on survival. The cases in whose specimens high VEGF expression was observed had poorer survival $(P<0.05)$.

\section{Analysis of Cox's proportional hazard reg- ression model}

After the analysis of univariables, the factors with a significance level of $P<0.05$ were analyzed using the univariate Cox's proportional hazard regression model. These factors were entered into the multivariate Cox's model. Independent of pathological subtype, TNM stage, perineural-invasion, recurrence, surgical modality, chemotherapy, radiotherapy, expression of NGF and VEGF were prog- 
nostic for survival in SACC patients (Tables 3, 4).

\section{Discussion}

SACC is an unpredictable neoplasm in its evolution and behaviors as it grows slowly, but tends to produce perineural invasion and vascular metastasis in early stages, in which highlyexpressed growth factors or their receptors such as TGF, EGFR have been shown to be significant mediators (Chiang et al., 2001; Gibbons et al., 2001; Liu et al., 2007). However, the mechanism of the malignancy's propensity to invade and spread along nerves and blood vessels still remains unclarified. Meanwhile, which factors contribute to the worse prognosis of SACCs remains controversial.

Table 3 Univariate Cox regression analysis of the prognostic factors

\begin{tabular}{lccc}
\hline \multicolumn{1}{c}{ Risk factor } & $\begin{array}{c}\text { Regression } \\
\text { coefficient }\end{array}$ & $\begin{array}{c}\text { Relative } \\
\text { risk }\end{array}$ & $P$ \\
\hline Site of origin & 0.154 & 6.023 & 0.003 \\
Pathological subtype & 3.236 & 3.422 & 0.043 \\
TNM stage & 1.283 & 2.804 & 0.045 \\
Perineural-invasion & 0.242 & 0.103 & 0.042 \\
Recurrence & 2.316 & 7.401 & 0.007 \\
Surgical modality & -8.168 & 3.343 & 0.037 \\
Radiotherapy & -10.128 & 0.022 & 0.029 \\
Chemotherapy & -11.049 & 0.031 & 0.011 \\
NGF & 1.147 & 6.559 & 0.026 \\
VEGF & 0.115 & 4.226 & 0.011 \\
\hline
\end{tabular}

Table 4 Multivariate Cox regression analysis of the prognostic factors

\begin{tabular}{lccc}
\hline \multicolumn{1}{c}{ Risk factor } & $\begin{array}{c}\text { Regression } \\
\text { coefficient }\end{array}$ & $\begin{array}{c}\text { Relative } \\
\text { risk }\end{array}$ & $P$ \\
\hline Pathological subtype & 0.213 & 0.138 & 0.017 \\
TNM stage & 1.382 & 1.553 & 0.046 \\
Perineural-invasion & 9.279 & 1.785 & 0.002 \\
Recurrence & 7.010 & 7.863 & 0.008 \\
Surgical modality & -8.550 & -1.311 & 0.011 \\
Radiotherapy & -11.134 & -2.214 & 0.014 \\
Chemotherapy & -10.642 & -1.136 & 0.023 \\
NGF & 6.288 & 1.124 & 0.037 \\
VEGF & 7.741 & 1.069 & 0.044 \\
\hline
\end{tabular}

In accordance with other reports, we found SACC of the major salivary glands more frequently originated in the submandibular and sublingual glands, with the prevalence of minor salivary glands as the primary tumor site (Garden et al., 1995; van der Wal et al., 2002; Kokemueller et al., 2004). It is often believed that with decreasing size of salivary gland of origin, tumors are more likely to be malignant, portending a worse prognosis. In our study, while site of origin was significant in the univariate analysis, it was not significant in the multivariate analysis. The reason for this may be that the population size was not large enough to demonstrate a significant difference.

For pathological subtype, a great many authors have addressed subtype effect on the clinical outcome of ACCs (Chummun et al., 2001; Sung et al., 2003; Zhu et al., 2003). We also observed that the cribriform-tubular was the pathological subtype associated with a higher postoperative survival in the included cases, and it was represented as one of the independent determining factors for the prognosis in the Cox regression analysis, but the reason for this is unknown.

Previous published series have validated the independent role of TNM stage as a prognostic factor in ACC patients (Kokemueller et al., 2004; Perez et al., 2006). Similarly, we found SACCs of advanced TNM stage tended to have increased cancer-related mortality: TNM stage was an independent predictor of prognosis.

Some have found that perineural invasion portends a worse prognosis (Garden et al., 1995; van der Wal et al., 2002; Kokemueller et al., 2004); yet others find that perineural involvement does not impact the outcome (Sur et al., 1997). In our study, the cases with perineural invasion had lower survival rates, and it was an independent predictor of survival.

The presence of recurrence, in this retrospective study, led to a significantly worse outcome. In another literature, however, recurrence was not shown to have an obvious impact on survival (Rhee et al., 2006). Possibly, as a result of sample size, we did not have the power to detect this effect, and future studies to clarify its influence are needed.

To date, surgery is still the cornerstone of the 
treatment of SACC. In general, neck dissection can decrease the risk of metastatic diseases and improve the clinical outcome, but given the propensity for vascular invasion instead of lymph node metastases, the necessity of neck dissection is not categorical in SACCs. It was seen in this study, however, that patients who underwent extended resection and ipsilateral modified neck dissection had higher postoperative survival compared to those who underwent extended resection alone. The possible reason is that SACC cells could spread to cervical tissue through blood vessels, where new lesions would develop, then contributing to more distant metastases; and/or the dissection might reduce the tumor cells in the neck, helping to decrease the risk of developing metastatic diseases. Surgical resection with postoperative adjuvant chemotherapy or radiotherapy was the main therapeutic modality for SACC. Yet, the use of adjuvant radiotherapy remains controversial. It has been recommended for advanced-stage patients with positive margins, whereas others have advocated for this therapeutic option in all patients (Silverman et al., 2004; Mendenhall et al., 2004). In our study, the results of both univariate and multivariate analyses provided a rational for using postoperative radiotherapy to improve survival after surgery. Based on experience with other head and neck tumors, some investigators believe that concomitant chemoradiotherapy represents an alternative for patients with advanced disease (Aguilar-Ponce et al., 2004); however, according to other data, chemotherapy is relatively inefficacious in the treatment of ACC which grows slowly, and the agents such as 5-fluorouracil appear to have low rates of response (Haddad et $a l ., 2006)$. We used postoperative chemotherapy in 15 cases, and found it could significantly improve the survival rate.

There is increasing evidence showing that overexpression of NGF results in enhanced invasiveness of a variety of cancer cells and a worse prognosis (Fuminori et al., 2001; Zhu et al., 2002; Tsunoda et al. 2006). Moreover, previous studies found that NGF and its receptor were expressed in ACC tissue, and the expression levels were not the same between the solid and the cribriform-tubular type which was considered less aggressive, compared with the former (Sung et al., 2003; Zhu et al., 2003). Our results also showed that the immunoreactivity of NGF was relatively strong in SACC cells, with the positive rate higher in the solid than in the cribriform-tubular type, suggesting that SACC cells expressed NGF and the staining intensity correlated with pathological subtypes of different malignancy. Meanwhile, we observed that there was obvious heterogeneity in NGF expression between the perineural-invasion and the non-perineural-invasion groups, suggesting that it was associated with neurotropism. The probable explanation is that NGF, expressed by ACC cells, is attracted by NGF receptors in nerve tissues, causing tumor cells and nerves to grow together. In addition, NGF expression induces more neovascularization around neurofibers, which supplies nutrients for tumor growth, promoting faster proliferation of the tumor cells around and perineural invasion (Zhu et al., 1999; Zhu et al., 2003; Guo et al., 2006). In this study, the NGF positive rate was higher in the advanced-stage and the recurrence groups than in the early-stage and the recurrence-free groups, suggesting that in the procession of SACC, more NGF could be expressed, and invasiveness of tumor cells enhanced. Other than the explanation above, another proposed mechanism is that NGF promotes invasion and metastasis of neoplasms through mediating dephosphorylation of serine in actin-binding proteins leading to tumor cell movement, as well as stimulating tumor cells to produce more heparanase degrading heparan sulfate proteoglycan which is substantial in extracellular matrix (Marchetti and Nicolson, 1997; Walch and Marchetti, 1999; Shonukan et al., 2003). Our analyses demonstrated that the pro-gnosis of non-perineural-invasion, recurrence-free, cribriform-tubular and early-stage patients was better in those whose specimens NGF level was lower. In multivariate analysis, the partial regression coefficient of NGF was 6.288, indicating that the prognosis of patients with high NGF expression differed significantly from that of those who lacked it: as NGF expression was elevated, the mortality risk of SACCs increased.

The overexpression of VEGF and its receptors has been detected in many malignancies and it is relevant to tumor invasion and metastasis. As a marker of potential clinical value, an elevated VEGF expression is connected with adverse out- 
come in several forms of cancer (Kourembanas et al., 1993; Witte et al., 1998; Lee et al., 2000; Sun et al., 2006; Schoenleber et al., 2009). In addition, it has been proposed that the prognosis of SACC patients is affected by pathological subtypes and the solid type present with distant metastases more frequently (Sung et al., 2003). It was observed in our study that there was more VEGF-immunoreactivity in SACC tissue, with the expression rate lower in the cribriform-tubular, the recurrence-free and the early-stage groups than in the solid, the recurrence and the advanced-stage groups. Moreover, the survival of the patients in the latter 3 groups was lower, in agreement with previous studies (Garden et al., 1995; Kokemueller et al., 2004; Mendenhall et al., 2004). Cox regression analysis also indicated that as TNM stage and VEGF expression levels increased, the prognosis worsened. The explanation may be that strong expression of VEGF can stimulate angiogenesis in tumor tissue and induce endothelium to secrete collagenase leading to the degradation of basal membrane; furthermore, due to the high permeability of new vessels, plasma protein is extravasated, providing stroma and nutrients for tumor growth and more neovascularization, which can promote tumor cells to achieve vascular invasion and distant spread (Liang et al., 2009). More importantly, our study showed that staining of VEGF was more intense in the perineuralinvasion than in the non-perineural-invasion group, the distribution of which was similar to that of NGF. Still, the correlation between these two factors in SACC tissues requires further study.

We discovered that NGF and VEGF expression increased in the specimens of SACC patients with invasion and metastasis. Elevated expressions of the two factors were independent predictors of impaired prognosis. Our findings suggest that NGF and VEGF are associated with invasion and metastasis of SACC; they can serve as criteria for assessing the prognosis of these patients.

\section{Conclusion}

In summary, staining intensity of NGF and VEGF was stronger in the specimens of perineuralinvasion, recurrence, high-malignancy pathological type and advanced TNM stage. Additionally, expressions of these two factors, as well as some pathoclinical factors like perineural invasion, recurrence, pathological subtype, TNM stage and treatment modality, were independent prognostic factors. The results suggest that NGF and VEGF significantly correlate with SACC invasion and metastasis, and they may serve not only as prognostic factors, but also as novel therapeutic targets in SACC. Nevertheless, more prospective work is needed to investigate the mechanism of how these two factors affect the progression of SACC. Moreover, further studies on the interaction between the two factors regarding invasion and metastasis are warranted.

\section{Acknowledgements}

This work was supported by National Natural Science Foundation of China (30060082), Scientific Research Foundation for the Returned Overseas Chinese Scholars, State Education Ministry ([2003] 593), Key Research Project Foundation of Guangxi Health Bureau (200006), Guangxi Science Foundation for Returned Overseas Scholars (0836013), Educational Scientific Research Foundation of Chinese Society of Higher Education (06AIL077 0110) and Innovation Project of Guangxi Graduate Education (2009105981003M174).

\section{References}

Aguilar-Ponce JL, Granados-García M, Villavicencio V, Poitevin-Chacón A, Green D, Dueñas-González A, et al. (2004). Phase II trial of gemcitabine concurrent with radiation for locally advanced squamous cell carcinoma of the head and neck. Ann Oncol, 15(2): 301-306.

Barnes L, Eveson JW, Reichart P, Sidransky D (2005). World Health Organization Classification of Tumours, Pathology \& Genetics, Head and Neck Tumours. Lyon: IARC Press, 221-222.

Burian M, Quint C, Neuchrist C (1999). Angiogenic factors in laryngeal carcinomas: do they have prognostic relevance? Acta Otolaryngol, 119(2): 289-292.

Chiang CP, Chen CH, Liu BY, Sun A, Leu JS, Wang JT (2001). Expression of transforming growth factor-alpha in adenoid cystic carcinoma of the salivary gland. $J$ Formos Med Assoc, 100(7): 471-477. 
Chummun S, McLean NR, Kelly CG, Dawes P, Fellows S, Meikle D, et al. (2001). Adenoid cystic carcinoma of the head and neck. Br J Plastic Surg, 54(6): 476-480.

Fanburg-Smith JC, Miettinen M (2001). Low affinity nerve growth factor receptor $(\mathrm{p} 75)$ in dermatofibrosareoma protuberans and other nonneural tumors: a study of 1,150 tumors and fetal and adult mormal tissues. Hum Pathol, 32(9): 976-983.

Fuminori S, Hiromitsu M, Takeo N, Yoshitsugu F, Toshitaka S, Sadaaki S, et al. (2001). Autocrine expression of neurotrophins and their receptors in prostate cancer. Int J Urol, 8(7): 28-34.

Garden AS, Weber RS, Morrison WH, Kianang K, Peters LJ (1995). The influence of positive margins and nerve invasion in adenoid cystic carcinoma of the head and neck treated with surgery and radiation. Int $J$ Radiat Oncol Biol Phys, 32(3): 619-626.

Gerwins P, Sköldenberg E, Claesson-Welsh L (2000). Function of fibroblast growth factors and vascular endothelial growth factors and their receptors in angiogenesis. Hepatology, 34(3): 185-194.

Gibbons MD, Manne U, Carroll WR, Peters GE, Weiss HL, Grizzle WE (2001). Molecular differences in mucoepidermoid carcinoma and adenoid cystic carcinoma of the major salivary glands. Laryngoscope, 111(8): 13731378.

Guo F, Lv CT, Li BP, Gao AZ, Chen Y (2006). Experimental study on the role of nerve growth factor in neural invasion of adenoid cystic carcinoma. J Oral Maxillofac Surg, 16(2): 106-120.

Haddad RI, Posner MR, Busse PM, Norris CM, Goguen LA, Wirth LJ, et al. (2006). Chemoradiotherapy for adenoid cystic carcinoma. Preliminary results of an organ sparing approach. Am J Clin Oncol, 29(2): 153157.

Inoue K, Ozeki Y, Suganuma T, Sugiura Y, Tanaka S (1997). Vascular endothelial growth factor expression in primary esophageal squamous cell carcinoma. Association with angiogenesis and tumor progression. Cancer, 79(2): 206-213.

Ishigami SI, Arii SS, Furutani M, Niwano M, Harada T, Mizumoto M, et al. (1998). Predictive value of vascular endothelial growth factor (VEGF) in metastasis and prognosis of human colorectal cancer. $\mathrm{Br} J$ Cancer, 78(10): 1379-1384.

Kan K, Yoshihisa Y, Katruhiro O (2002). Production of nerve growth factor by mouse hepatocellular carcinoma cells and Expression of TrkA in tumor-associated arteries in mice. Gastroenterology, 122(1): 1978-1986.
Kokemueller H, Eckhardt A, Brachvogel P, Hausamen JE (2004). Adenoid cystic carcinoma of the head and neck: a 20 year experience. Int J Oral Maxillofac Surg, 33(1): 25-31.

Kolokythas A, Cox DP, Dekker N, Jordan RCK, Schimdt BL (2006). Expression of nerve growth factor and tyrosine kinase a receptor in oral squamous cell carcinoma. Arch Otolaryngol Head Neck Surg, 132(8): 101-106.

Kourembanas S, McQuillan LP, Leung GK, Faller DV (1993). Nitric oxide regulates the expression of vasoconstrictors and growth factors by vascular endothelium under both normoxia and hypoxia. J Clin Invest, 92 (1): 99-104.

Lee JC, Chow NH, Wang ST, Huang SM (2000). Prognostic value of vascular endothelial growth factor expression in colorectal cancer patients. Eur J Cancer, 36(6): 748753

Liang J, You YH, Ouyang KX, Wang YP, Zhang HW (2009). Expression of vascular endothelial growth factor and microvessel density in salivary adenoid cystic carcinoma. Chin J Stomatol Res (Electronic Edition), 3(1): 23-26.

Liu L, Cai YL, Lv Y, Su LX (2007). Expression and significance of EGFR in salivary gland adenoid cystic carcinoma. J Oral Maxillofac Surg, 17 (4): 320-323.

Luo XL, Lv CT, Zhou ZH, Liu J (2006). Influence of nerve growth factor on the proliferation and apoptosis of adenoid cystic carcinoma cell line. J Oral Maxillofac Surg, 16(3): 205-208.

Maeda K, Chung YS (1996). Prognostic value of vascular endothelial growth factor expression in gastric carcinoma. Cancer, 77 (4): 858-863.

Marchetti D, Nicolson GL (1997). Human melanoma cell invasion: selected neurotrophin enhancement of invasion and heparanase activity. J Investig Dermatol Symp Proc, 2 (1): 99-105.

Mendenhall WM, Morris CG, Amdur RJ, Werning JW, Hinerman RW, Villaret DB (2004). Radiotherapy alone or combined with surgery for adenoid cystic carcinoma of the head and neck. Head Neck, 26 (2):154-162.

Mineta H, Miura K, Ogino T, Takebayashi S, Misawa K, Ueda Y, et al. (2000). Prognostic value of vascular endothelial growth factor (VEGF) in head and neck squamous cell carcinomas. Br J Cancer, 83(6): 775781.

Perez DE, Alves FA, Nishimoto IN, Almeida OP, Kowalski LP (2006). Prognostic factors in head and neck adenoid cystic carcinoma. Oral oncol, 42(2): 139-146. 
Rhee CS, Won TB, Lee CH, Min YG, Sung MW, Kim KH, et al. (2006). Adenoid cystic carcinoma of the sinonasal tract: treatment results. Laryngoscope, 116(6): 982-986.

Schoenleber SJ, Kurtz DM, Talwalkar JA, Roberts LR, Gores GJ (2009). Prognostic role of vascular endothelial growth factor in hepatocellular carcinoma: systematic review and meta-analysis. Br J Cancer, 100(9): 13851392.

Shonukan O, Bagayogo I, McCrea P, Chao M, Hempstead B (2003). Neurotrophin-induced melanoma cell migration is mediated through the actin-bundling protein fascin. Oncogene, 22(23): 3616-3623.

Silverman DA, Carlson TP, Khuntia D, Bergstrom RT, Saxton J, Esclamado RM (2004). Role for postoperative radiation therapy in adenoid cystic carcinoma of the head and neck. Laryngoscope, 114(7): 1194- 1199.

Sobin LH, Gospodarowicz MK, Wittekind CH (2010). International Union Against Cancer (UICC), TNM Classification of Malignant Tumours. Chichester: Blackwell Publishing Ltd, 22-57.

Sortino MA, Condorelli F, Vancheri C, Chiarenza A, Bernardini R, Consoli U, et a1. (2000). Mitogenic effect of nerve growth factor (NGF) in LNCaP prostate adenocarcinoma cells: role of the high and low affinity NGF receptors. Mol Endocrinol, 14(1): 124-136.

Sun DW, Wang YJ, Kong WJ, Liu BH (2006). Prognostic Value of Ki67 and VEGF Expression in Laryngeal Squamous Cell Carcinoma. Chin J Clinic Oncol, 3(1): 20-24.

Sung MW, Kim KH, Kim JW, Min YG, Seong WJ, Roh JL, et al. (2003). Clinicopathological predictors and impact of distant metastasis from adenoid cystic carcinoma of the head and neck. Arch Otolaryngol Head Neck Surg, 129(11): 1193-1197.

Sur RK, Donde B, Levin V, Pacella J, Kotzen J, Cooper C, et al. (1997). Adenoid cystic carcinoma of the salivary glands: a review of 10 years. Laryngoscope, 107(9): 1276-1280.

Tsunoda S, Okumura T, Ito T, Mori Y, Soma T, Watanabe G, et al. (2006). Significance of nerve growth factor overexpression and its autocrine loop in oesophageal squamous cell carcinoma. Br J Cancer, 95(3): 322-330.

Umeda M, Komatsubara H, Nishimatsu N, Oku N, Shibuya

Y, Yokoo S, et al. (2002). Establishment and characterization of a human adenoid cystic carcinoma line of the salivary gland which is serially transplantable and spontaneously metastasises to the lung in nude mice. Oral Oncol, 38(1): 30-34.

van der Wal JE, Becking AG, Snow GB, van der Waal I (2002). Distant metastases of adenoid cystic carcinoma of the salivary glands and the value of diagnostic examinations during follow-up. Head Neck, 24(8): 779-783.

Volm M, Koomagi R, Mattern J (1997). Prognostic value of vascular endothelial growth factor and its receptor Flt-1 in squamous cell lung cancer. Int $J$ cancer, 74(1): 64-67.

Walch ET, Marchetti D (1999). Role of neurotrophins and neurotrophins receptors in the in vitro invasion and heparanase production of human prostate cancer cells. Clin Exp Metastasis, 17(4): 307-314.

Witte L, Hicklin DJ, Zhu Z, Pytowski B, Kotanides H, Rockwell P, et al. (1998). Monoclonal antibodies targeting the VEGF receptor-2 (FIk-1/KDR) as an anti-angiogenic therapeutic strategy. Cancer Metastasis Rev, 17(2): 155-161.

Xu WG, Yang GL, Zhou LX, Xie YQ, Zhang LJ (2004). Prognostic value of VEGF expression in primary esophageal squamous cell carcinoma. Chin $J$ cancer Res, 16(2): 85-89.

Zhu WQ, Ji XR, Zhou FT, Li NY (2003). Expression of NGF, p75 and TrKA in adenoid cystic carcinoma of salivary glands. J Modern Stomatol, 17(2): 104-108.

Zhu Z, Friess H, diMola FF, Zimmermann A, Graber HU, Korc M, et al. (1999). Nerve growth factor expression correlates with perineural invasion and pain in human pancreatic cancer. J Clin Oncol, 17(8): 2419-2428.

Zhu Z, Kleeff J, Kayed H, Wang L, Korc M, Büchler MW, et al. (2002). Nerve growth factor and enhancement of proliferation, invasion, and tumorigenicity of pancreatic cancer cells. Mol Carcinog, 35(3):138-147.

\footnotetext{
*Corresponding authors: Xiao-lin Nong

Address: Department of Oral and Maxillofacial Surgery, College of Stomatology, Guangxi Medical University, Nanning 530021, China

Tel: $867715358045 \quad$ E-mail: lab.nong@gmail.com
} 